\title{
EFSUMB COVID webinars
}

EFSUMB WEBINAR

Tuesday 14 April, 202017.30 CEST Lung Ultrasound for the Covid-19 era

if you missed the live presentations visit the webinar archive

http://www.efsumb.org/blog/webinar-archive-2

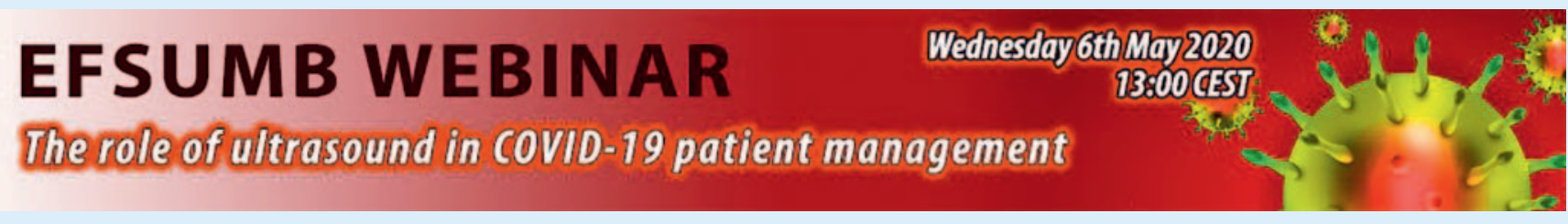

- lung ultrasound examination protocol for assessment of COVID-19 patients: Konrad Stock

- COVID-19 outbreak - the value of lung ultrasound for clinical decision making: Alessandro Zanforlin

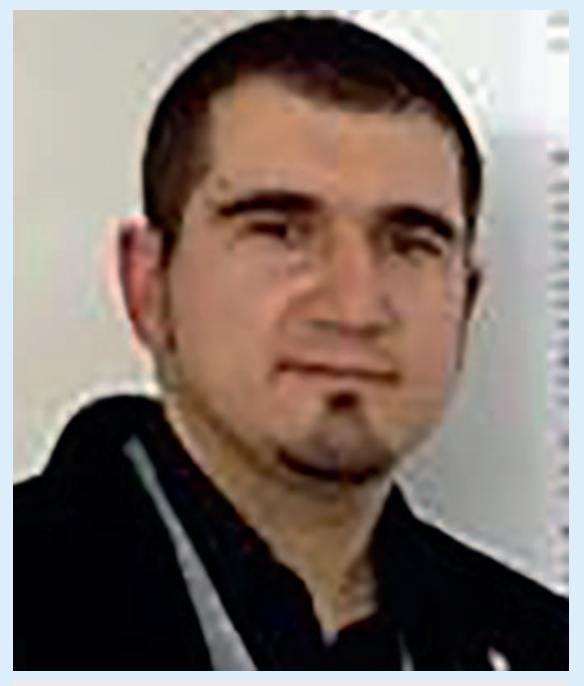

Dr Alessandro Zanforlin
Dr Alessandro Zanforlin: Mobile Lung ultrasound Unit, Radiodiology service, Bolzano hospital, Pneumological Service of South Tyrol writes

My interest on Chest ultrasound began in 2005 as I took part in the first Chest Ultrasound course for pulmonologists that took place in Bologna, then I completed the SIUMB general US theoretical/practical educational path.

After completing the specialisation in pulmonology, I worked as a pulmonologist in Internal medicine ward from 2009 to February 2020 with special attention on research on clinical applications of chest ultrasound: pleural disease, diaphragm motion analysis, lung diseases and began since 2011 the collaboration with italian network of chest ultrasound performed that in the last years is known as "Accademia di Ecografia Toracica (AdET)" Chest Ultrasound Academy. Together, organising many US courses, we have contributed to the diffusion fo chest ultrasound in italy (documented by a recent survey amid italian pulmonologist that sowed large diffusion of the technique, in particular in Northern italy). Our network takes care of research on chest US and continuous education and knowledge sharing in particular through our facebook group (now it takes account of more than 3700 members) and national congresses (the last one took place right in Bolzano, my city on 25 January 2020, with 120 participants and many others more in overbooking...).

In February I was transferred to the pulmonology Service of the Province of Bolzano (respiratory outpatients). After the beginning of the COVID emergency in Bolzano, in order of my experience in Chest US, I was called back to the Hospital and engaged by the Radiology department as a "mobile lung ultrasound unit", with the aim of helping in technology assessment, teaching chest US to other colleagues (Emergency department, Intensive Care Unit in particular) and organising the Chest US activity in the hospital.

After searching around the hospital all the US machines that were unused or underused because of COVID emergency, we redistributed the US apparels that were needed to activate the COVID-triage screening in the emergency department and for the new-COVID departments (normal COVID ward and COVID-ICU), to provide US empowerment of these wards and limit the US-machine movement between wards that would have been dangerous in terms of contamination.

Then my daily activity was to perform the LungUS evaluation of COVID-ICU patients and monitor their lung condition, and perform the Lung-US screening in the ED. In addition of the conduction of US examinations, I took care to teach to the colleague the technique, so that some of them became progressively independent, contributing in covering the activity also in my absence. We have now collected precious informations about the use of chest US in those settings (ED and ICU) and we are now elaborating the data to share them with the scientific community. 
EFSUMB WEBINAR

8 April 2020, 18.00 CEST
CEUS in Liver Imaging

\section{Click for registration $>$}

\section{EFSUMB WEBINAR}

13 May 2020, 18.00 CEST

- percutaneous ablation of liver lesions (RFA, MW, IRE): Franca Meloni

- HIFU ablations of prostate lesions: Dirk Clevert

- abdominal interventions: Zeno Sparchez

\section{EFSUMB WEBINAR}

\section{September 2020, 11.00 CEST CEUS for the management of focal liver disease}

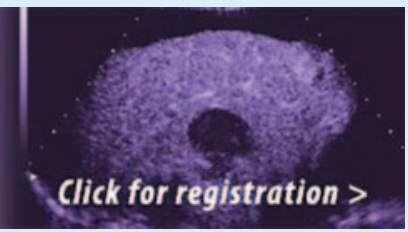

- clinical value of CEUS in ggllbladder disease: Xiang Fei

- CEUS diagnosis of new hepatic nodules in patients with colorectal cancer during chemotherapy: Guang-Jian Liu

- Combined application of navigation and contrast-enhanced ultrasound in liver tumor: Kai Li

\section{EFSUMB WEBINAR}

6 November 2020, 11.00 CET

- value of CEUS applications in thyroid diseases: Wen Luo

- Significance and value of CEUS in thyroid disease diagnosis and treatment: Hong Yang

- Application of contrast-enhanced ultrasound in differential diagnosis of complex gynaecology masses: Xinling Zhang

\section{EFSUMB WEBINAR}

\section{COMING}

SOON

\section{EFSUMB POSITION STATEMENT ON DERMATOLOGIC UITRASOUND:}

A KEYSTONE FOR A NEW APPLICATION OF ULTRASOUND IN DERMATOLOGY

\section{Euroson Schools}

Please visit the EFSUMB website for the revised School dates

http://www.efsumb.org/blog/euroson-schools 


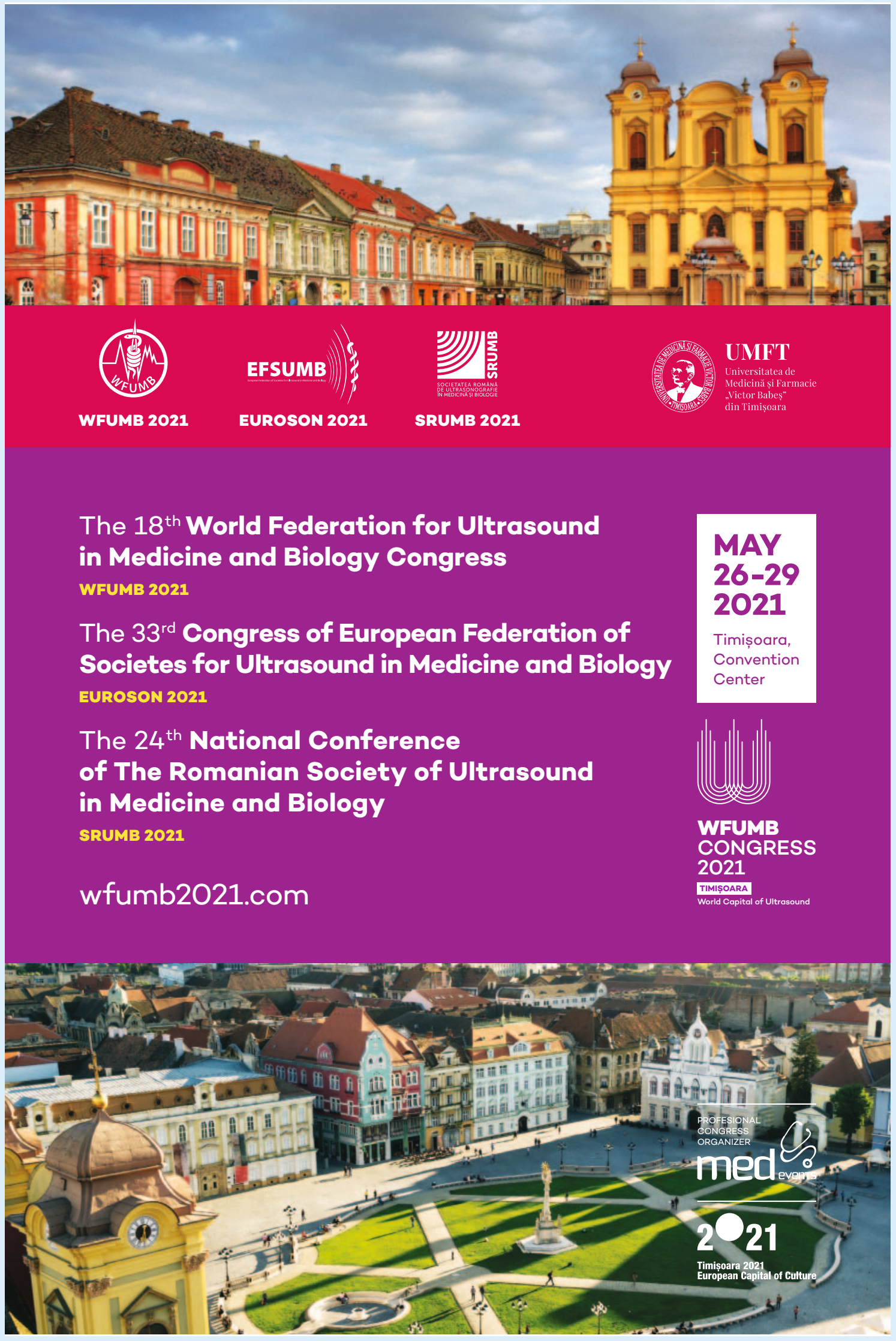



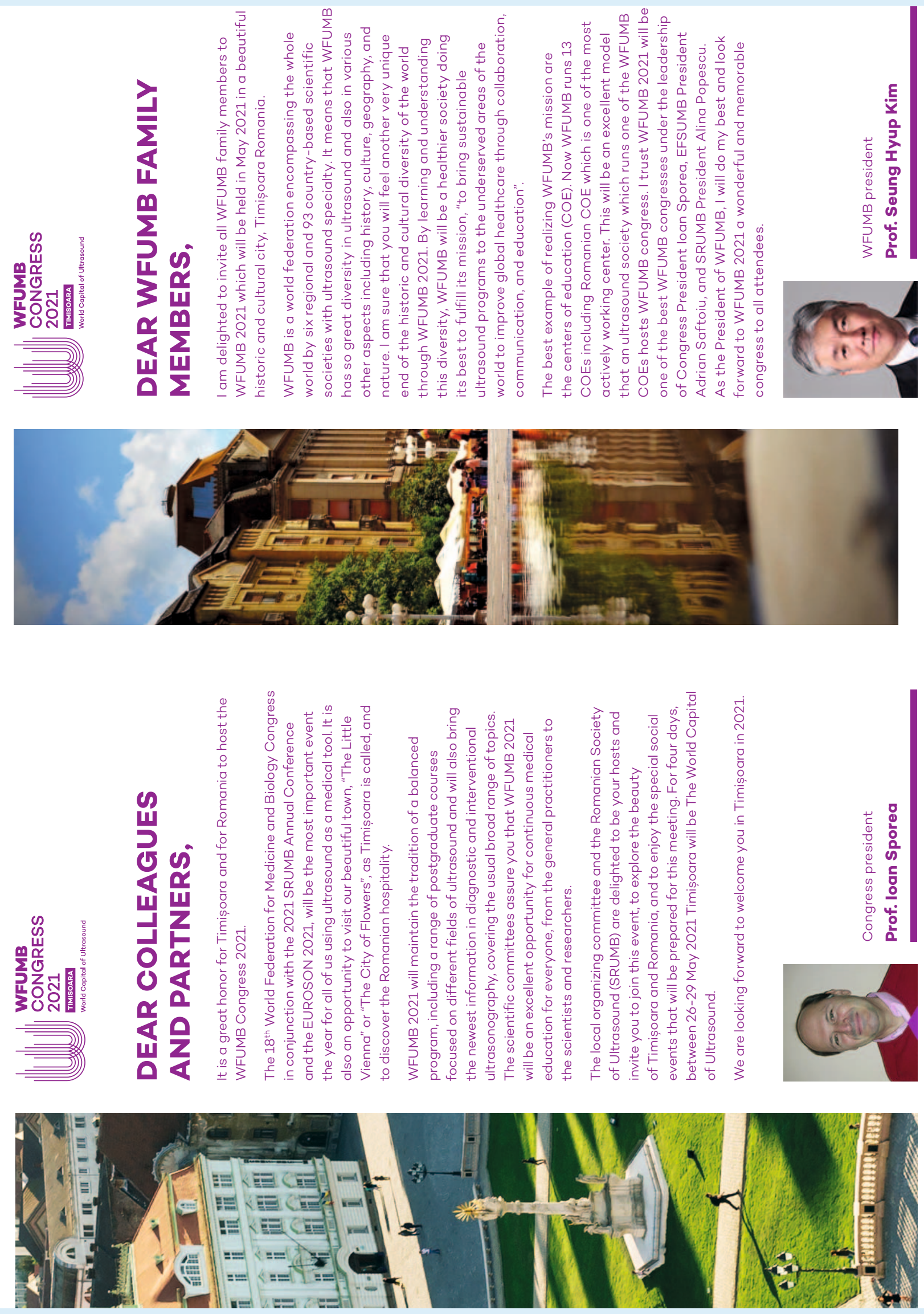

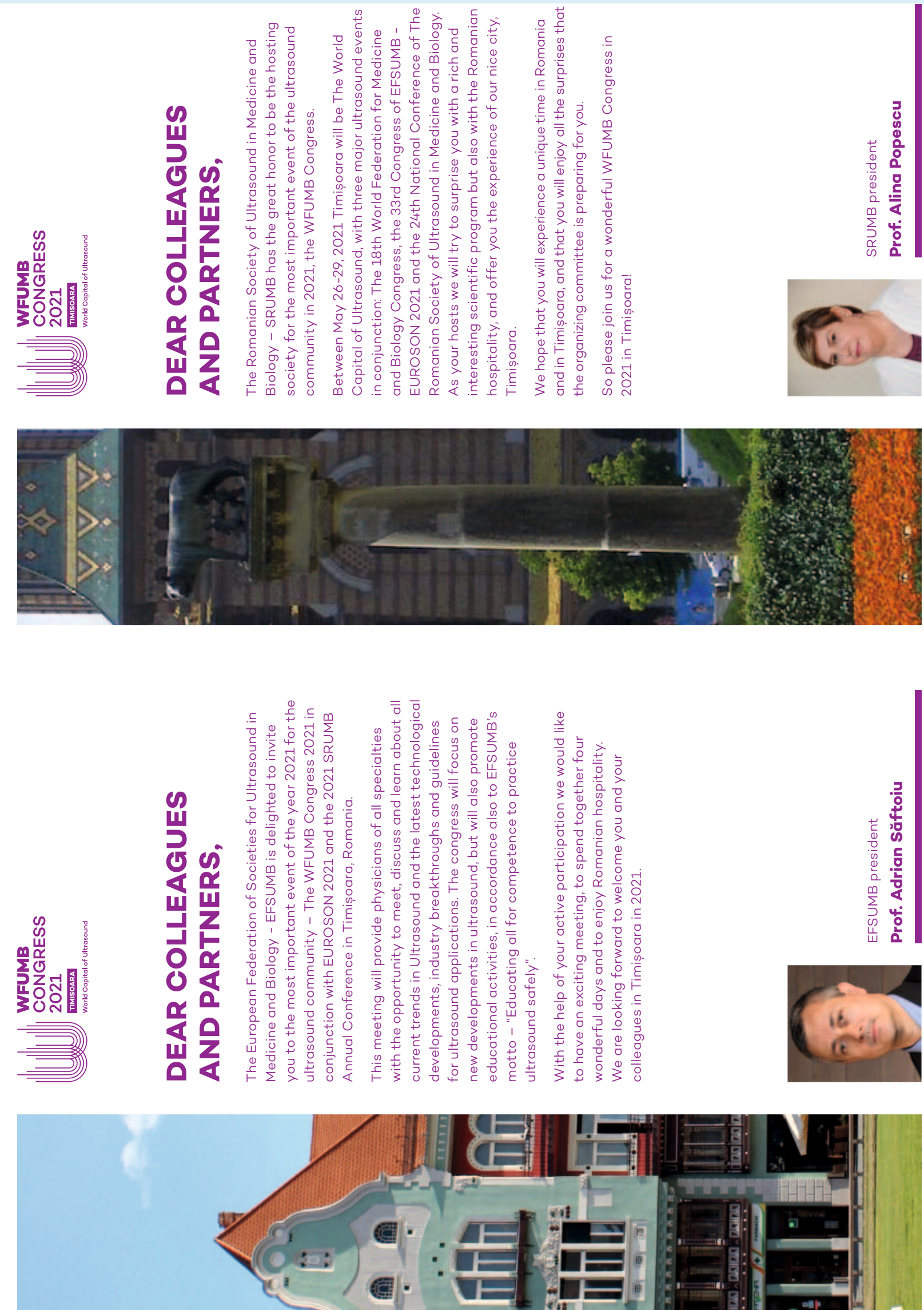

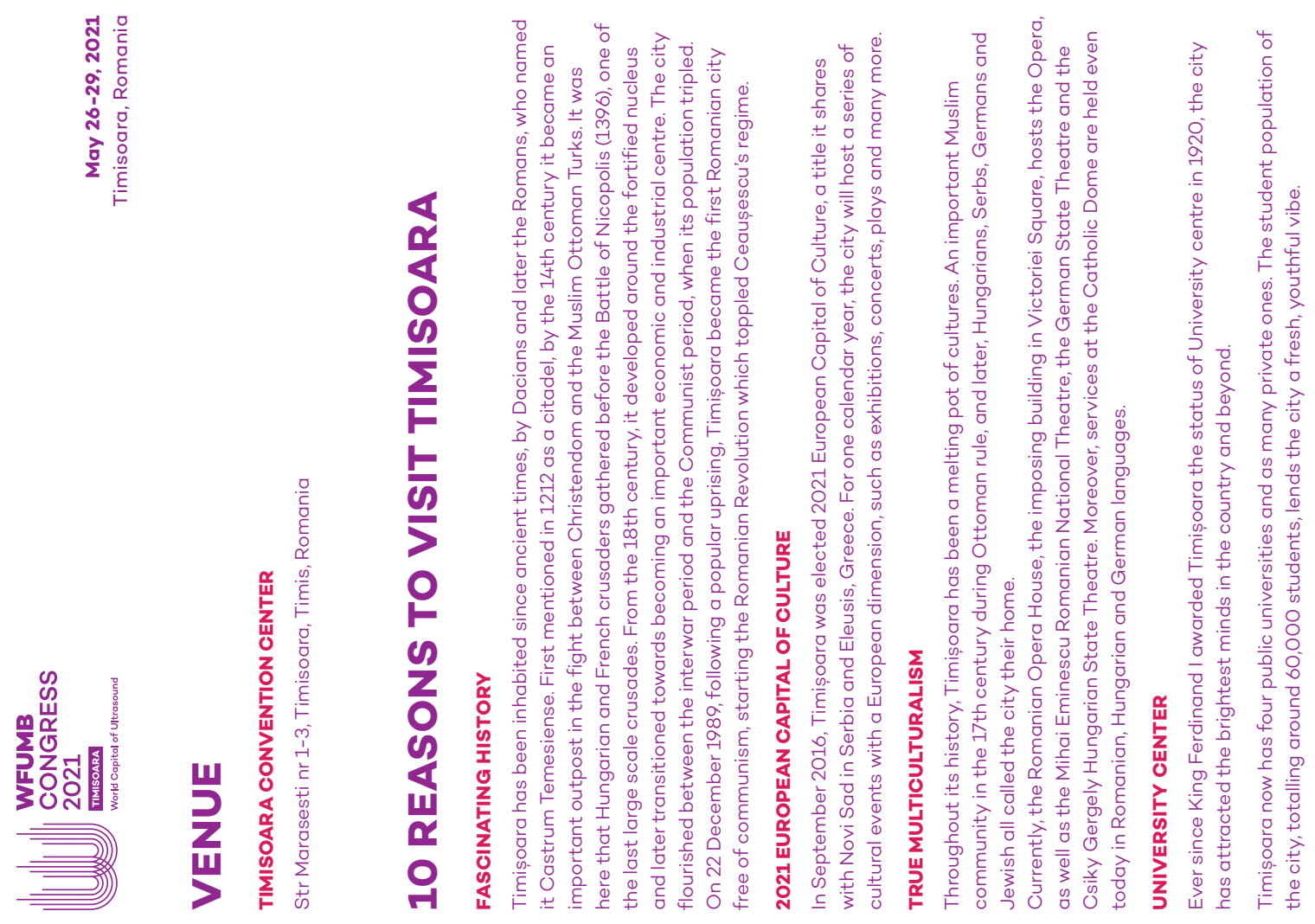

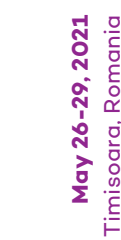
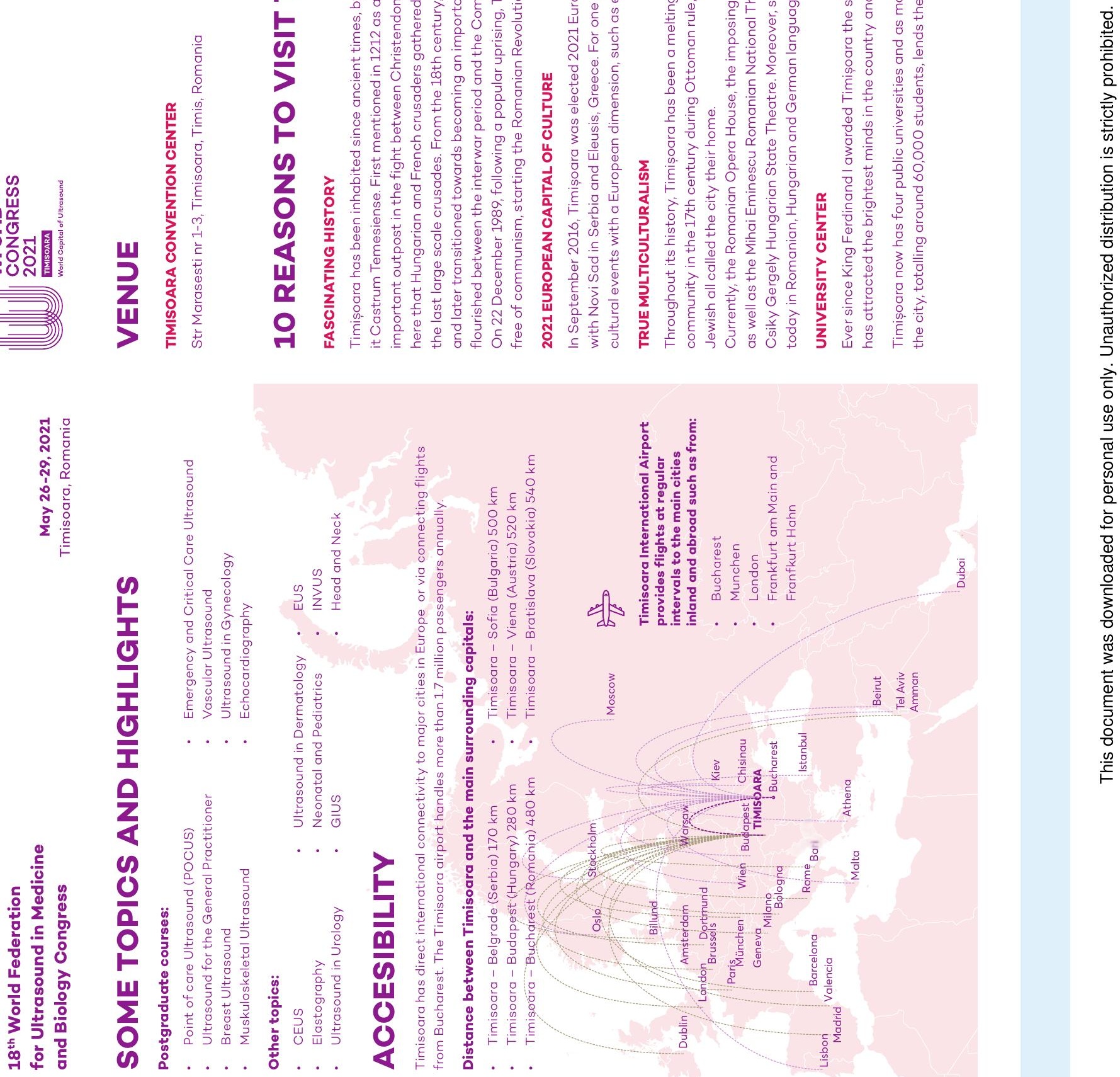

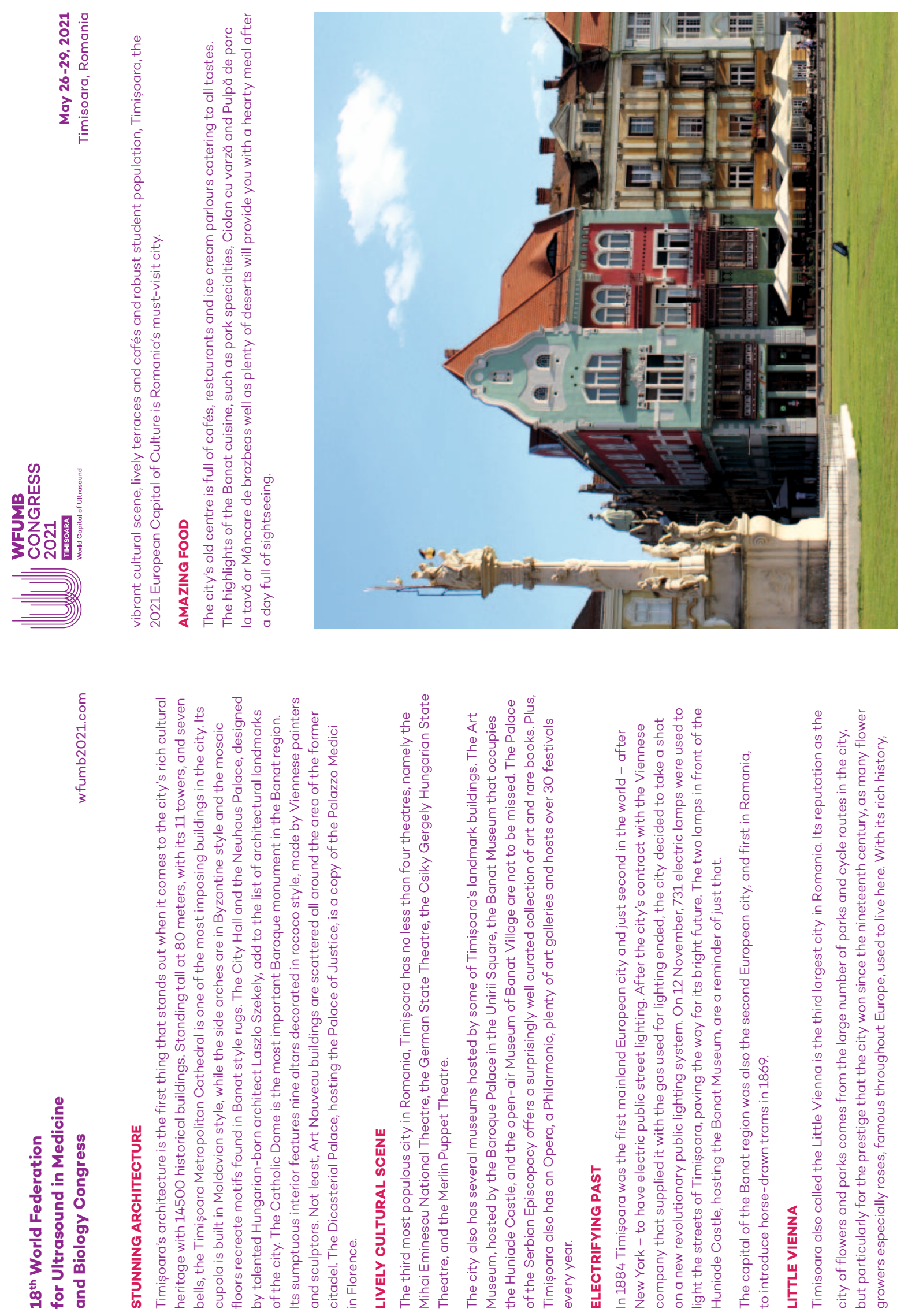

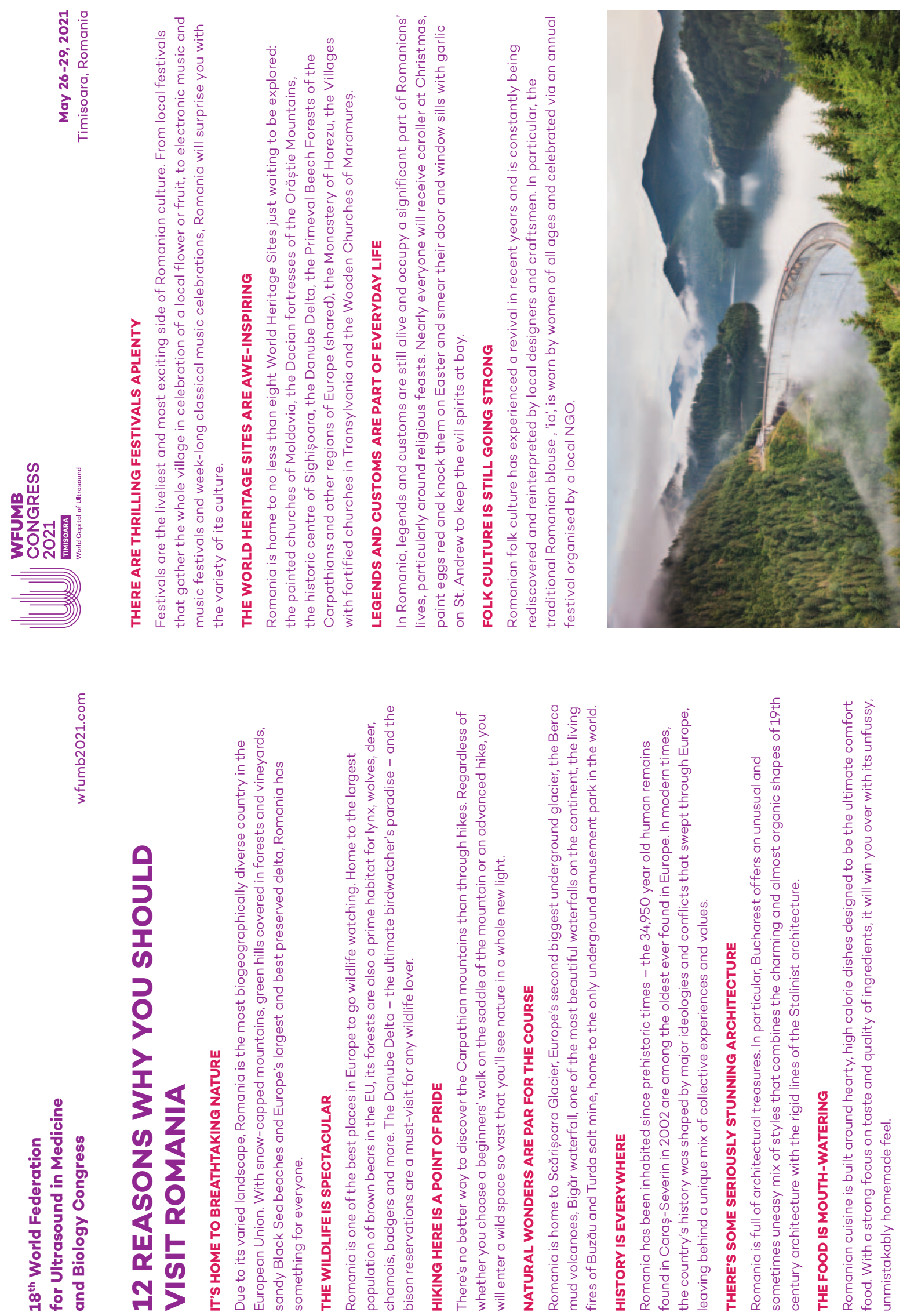


\section{REGISTRATION FEES}

\begin{tabular}{|c|c|c|c|}
\hline REGISTRATION TYPE & $\begin{array}{l}\text { EARLY } \\
\text { REGISTRATION } \\
\text { Before } 10.02 .2021\end{array}$ & $\begin{array}{l}\text { REGULAR } \\
\text { REGISTRATION } \\
\text { Until } 24.04 .2021\end{array}$ & $\begin{array}{l}\text { ON SITE } \\
\text { From } 25.04 \& \\
\text { On site }\end{array}$ \\
\hline $\begin{array}{l}\text { WFUMB/EFSUMB/SRUMB member } \\
\text { (Proof of membership is required) }\end{array}$ & 300 Euro & 400 Euro & 600 Euro \\
\hline Non member & 450 Euro & 550 Euro & 700 Euro \\
\hline Fellow in training (residents)/PhD students & 150 Euro & 250 Euro & 300 Euro \\
\hline $\begin{array}{l}\text { Medical Student } \\
\text { Only Wednesday or Thursday } 30 / \text { day }\end{array}$ & 70 Euro & 70 Euro & 100 Euro \\
\hline Sonographer & 200 Euro & 300 Euro & 400 Euro \\
\hline
\end{tabular}

\section{ONLINE REGISTRATION}

We are excited to announce that registration for the WFUMB congress will open on our website on March, 15 wfumb2021.com

\section{IMPORTANT DATES}

\section{DEC 2020}

Abstract submission

\section{FEB 2021}

Abstract acceptance notification

\section{FEB 2021}

Early bird registration fee

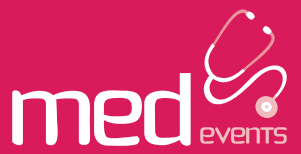

PROFESSIONAL CONGRESS ORGANIZER

phone +40364146681

fax +40 364108990

Cluj-Napoca 400012

3/3 Victor Babes Street

contact@medevents.ro

www.medevents.ro

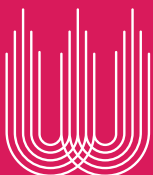

WFUMB CONGRESS 2021

TIMIȘOARA

World Capital of Ultrasound

wfumb2021.com 